\title{
Surface preparation and isotropic shear bond strength properties of superficial bovine dentin
}

\author{
Camila Sabatini $^{{ }^{*}}$, Sebastiano Andreana ${ }^{1}$, Zhe $\mathrm{Wu}^{2}$ \\ ${ }^{1}$ SUNY at Buffalo, School of Dental Medicine, Department of Restorative Dentistry, Buffalo, NY, USA \\ ${ }^{2}$ School of Stomatology, Jilin University, Changchun, China \\ Email: ${ }^{*}$ cs252@,buffalo.edu
}

Received 20 May 2013; revised 21 June 2013; accepted 10 July 2013

Copyright (C) 2013 Camila Sabatini et al. This is an open access article distributed under the Creative Commons Attribution License, which permits unrestricted use, distribution, and reproduction in any medium, provided the original work is properly cited.

\begin{abstract}
The effect of different types of surface preparation with $\mathrm{SiC}$ abrasive paper on the shear bond strength (SBS) of superficial bovine dentin obtained from the incisal, middle and cervical thirds were evaluated. Dentin substrates were obtained with twenty specimens for each location-grit combination. Superficial dentin was exposed and prepared to 120-, 320-, or 600-grit SiC; the dentin surfaces were treated with Optibond Solo Plus (Kerr) and polymerized for $20 \mathrm{~s}$. The specimens were placed in a jig, filled with resin composite Z100 (3M-ESPE), polymerized for $40 \mathrm{~s}$ according to manufacturer's instructions, and stored for $24 \mathrm{~h}$ at $37^{\circ} \mathrm{C}$ and $100 \%$ humidity. After $24 \mathrm{~h}$, SBS was measured using a loading testing machine (Ultradent) and expressed in megapascals. A two-way ANOVA and Tukey test were used for data analysis. No statistically significant effect of the location $(P=$ $0.254)$ or interaction grit-location $(P=0.629)$ were observed on SBS. Statistically significant effect of the grit on the SBS was detected $(P<0.001)$ with 320 -grit being statistically different from 600 -grit $(P=0.011)$ and 120-grit $(P<0.001)$. No significant differences were observed between 600-grit and 120-grit $(P=$ 0.413). Regardless of the location, 320-grit consistently showed the lowest SBS indicating that different surface grit preparations have an effect on dentin SBS values.
\end{abstract}

Keywords: Resin Composite; Dentin Adhesion; Bond Strength; Isotropic; Surface Preparation; Failure Mode

\section{INTRODUCTION}

Adhesion to tooth structure, particularly to dentin, has been subject of extensive research in the last few dec-

${ }^{*}$ Corresponding author. ades [1-3]. The success of adhesive restorations is largely a function of factors relative to the bonding system, substrate and composite. Furthermore, in-vitro bond strength tests incorporate additional variables relative to the sample preparation, testing itself and storage conditions that are also known to have an effect in the complex nature and interactions taking place at the adhesive interfaces [4] and they all deserve consideration when evaluating strength of these interfaces. Bond strength tests have been extensively used and play a paramount role in the screening of dental adhesives since they are relatively easy to perform, do not require much armamentarium and are able to provide immediate information regarding the strength, adhesive or cohesive, of the bonded interface. While bond strength tests have allowed the development of improved adhesive systems and techniques, they are subject to a number of limitations and its results vary considerably depending on a number of structural and testing procedures [5]. A set of guidelines was developed by the International Organization for Standardization (ISO) to standardize bond strength testing of adhesive interfaces and thus facilitate reproducibility of the testing conditions and allow comparisons among studies. (ISO/TR 11405:1994) However, despite standardization efforts, variability in the testing conditions still exists particularly in aspects related to the substrate and testing itself compromising the validity of the comparisons among studies [6].

Variables inherent to the substrate, particularly dentin, are especially critical due to the large morphological variability known to exist between different teeth and in various regions of the same tooth [7]. Bond strength studies commonly use human third molars or bovine incisors as a substrate for bonding. Despite some anatomical and permeability differences [8], histochemical and morphological studies have shown that human and bovine dentin are essentially the same [9-12]. Studies have reported no differences in the concentration of 
tubules per $\mathrm{mm}^{2}$ between coronal human and bovine dentin [12]. However, compared to human molars, bovine incisors dentinal tubules are of larger size and show more porous intertubular dentin [13]. According to Nakamichi, only superficial dentin may be considered a suitable substitute to human dentin [10]. Irrespective of the type of substrate used, most studies fail to provide critical information regarding aspects such as the specific location of the bonding and the potential effect that this location may have on the bond strength results. The anisotropic bond strength behavior of human dentin has been established in previous studies [14,15]. However, limited information is available regarding the anisotropic bond strength properties of bovine dentin.

Surface preparation of the substrate has also been shown to have an effect in the bond strength of adhesive interfaces $[16,17]$. Studies have evaluated the effect of different types of surface preparation on dentin surface roughness $[18,19]$. Carbide and diamond rotary cutting instruments and silicon carbide abrasive paper create rough surfaces that are different in topography and smear layer thickness [20]. Studies evaluating the effect of surface preparation on bond strength normally use carbide or diamond burs simulating a clinical situation [21,22]. However, current ISO standards for bond strength testing recommend surface preparation with 600-grit silicon carbide ( $\mathrm{SiC})$ abrasive paper. This method produces a relatively smooth surface that does not reproduce the surface topography and smear layer thickness obtained with rotary cutting instruments [20]. Studies have evaluated the effect of different surface preparation methods on the surface roughness [20], wettability [17] and thickness of smear layer [23,24]. However, most of these studies fail to provide critical information regarding specific location of bonding within the crown. The authors are not aware of studies evaluating the shear bond strength of surfaces prepared with different progressively higher grits of silicon carbide $(\mathrm{SiC})$ abrasive paper when bonded to different crown locations.

The objective of this study was to evaluate the effect of different types of surface preparation with silicon carbide $(\mathrm{SiC})$ abrasive paper on the Shear Bond Strength (SBS) of superficial coronal dentin obtained from the incisal, middle and cervical thirds of bovine incisors. The null hypotheses were: 1) there would be no difference in SBS values obtained from the incisal, middle and cervical thirds; 2) there would be no difference in SBS values after surface preparation with the different grits of abrasive paper.

\section{MATERIALS AND METHODS}

\subsection{Specimen Preparation}

Thirty non-carious bovine mandibular central incisors were used to obtain dentin substrates for bonding. The incisors were used within one month of extraction and stored in an aqueous disinfectant $(0.5 \%$ chloramine $\mathrm{T}$ solution at $4^{\circ} \mathrm{C}$ ) until ready to be used. A slow-speed saw (Isomet, Buehler, Lake Bluff, IL, USA) with a diamond disk under cooling water was used to separate the crowns from the roots at the CEJ level. From each crown, six sections were obtained by making one longitudinal section along the middle of the crown and three transverse sections between the crown's incisal, middle and cervical thirds. This yielded a total of 180 specimens with a sample size of twenty $(\mathrm{N}=20)$ for each location-grit combination. The sectioned specimens were embedded in a chemically-polymerized methacrylate (Fastray, HJ Bosworth, Skokie, IL, USA) with the facial surface exposed. The exposed surface was ground flat on a model trimmer until superficial dentin was revealed. Each group of sixty specimens including samples obtained from the incisal, middle and cervical thirds were polished to a final coarseness of 120-, 320- or 600-grit with silicon carbide $(\mathrm{SiC})$ abrasive paper (Buehler). The prepared specimens were stored in de-ionized water at $4^{\circ} \mathrm{C}$ until ready to be bonded.

\subsection{Shear Bond Strength Testing and Statistical Analysis}

Restoration of the different study groups was randomized to avoid bias relative to sequence of tooth restoration. One hour prior to bonding, the specimens were acclimatized to room temperature $\left(23^{\circ} \mathrm{C} \pm 2^{\circ} \mathrm{C}\right)$. Immediately before the start of the procedures, the specimen surfaces were slightly refinished with the same final coarseness of $\mathrm{SiC}$ paper used to prepare the surface initially in order to expose fresh dentin. Dentin specimens were etched with $35 \%$ phosphoric acid (Ultra-etch, Ultradent, South Jordan, UT, USA) for 15 seconds, rinsed and blot dried for moist bonding. An etch-and-rinse adhesive system (Optibond Solo Plus, Kerr, Orange, CA, USA) was applied and polymerized for 20 seconds following manufacturer's instructions with a light curing unit (Bluephase C8, Ivoclar-Vivadent, Amherst, NY, USA). A minimum power density of $800 \mathrm{~mW} / \mathrm{cm}^{2}$ was ensured by periodically monitoring the unit's output with a radiometer (Demetron, Kerr). The specimens were placed on a specially fabricated bonding jig (Ultradent) with a cylindrical mold of $2.38 \mathrm{~mm}$ in diameter. The mold was filled with resin composite (Z100, 3M-ESPE, Saint Paul, MN, USA) in a single increment no greater than $2 \mathrm{~mm}$ and polymerized for 40 seconds. Immediately after bonding, specimens were stored in an incubator at $37^{\circ} \mathrm{C}$ and $100 \%$ humidity for 24 hours.

Shear bond strength was measured using a testing machine (Ultratester, Ultradent) at a test speed of $1 \mathrm{~mm} /$ min with a load cell of $1000 \mathrm{lbs}(453.6 \mathrm{Kg})$. A notch- 
ed crosshead designed to match the diameter of the bonded specimen was used to apply the testing load. Specimens were stabilized in a testing jig which was free to move to facilitate positioning under the load. The test base was then positioned so that the notched crosshead was placed against the specimen surface and the notch was fitted to the bonded specimen. The load required to debond the specimen was recorded and expressed in megapascals ( $\mathrm{MPa})$ by dividing the load by the surface area of the bonded specimen. Shear bond strength values were recorded at 24 hours and the mean SBS values per study group were calculated. A two-way analysis of variance (ANOVA) was used to evaluate the effect of the crown location and surface preparation on shear bond strength. Pairwise multiple comparison procedures $(\mathrm{Tu}-$ key test) was used to identify these differences among the individual groups. A significance level of 0.05 was used for all tests.

\subsection{Analysis of the Mode of Failure}

Mode of failure was analyzed by field emission scanning electron microscope (FE-SEM) (Hitachi SU-70, Hitachi, Krefeld, Germany) observation of the dentin-adhesive interface in backscattered electron mode. The assessment was conducted by one trained examiner (CS). Both sides of the fractured interface of three representative specimens for each location-grit combination was observed and scored. The highest value, a value close to the mean and the lowest bond strength value were subjected to SEM analysis at a magnification of $50 \times$. The orientation of the dentinal tubules for the different crown thirds and the surface topography obtained after preparation with the different grits of abrasive paper was confirmed by SEM observation of additional dentin surfaces in secondary electron mode. The specimens were desiccated, and coated with a $20 \mathrm{~nm}$ layer of evaporated carbon to provide conductivity.

The fractured surfaces were classified into one of five failure modes: 1) cohesive in dentin; 2) adhesive at the interface between dentin and adhesive resin; 3 ) cohesive in adhesive resin; 4) adhesive at the interface between the adhesive resin and composite; 5) cohesive in composite; 6) mixed failure across the different layers of the interface.

\section{RESULTS}

The mean SBS values obtained for the different location-grit combinations are shown in Figure 1 and Table 1. Table 2 summarizes the two-way ANOVA results. Statistically significant effect of the surface preparation on the SBS ( $\mathrm{P}<0.001)$ was detected with 320-grit being statistically lower than 600 -grit $(\mathrm{P}=0.011)$ and 120 -grit $(\mathrm{P}<0.001)$. No significant differences were observed between 600-grit and 120-grit $(\mathrm{P}=0.413)$. No statistically significant effect of the location variable $(\mathrm{P}=0.254)$ or the interaction grit-location on the SBS was detected $(\mathrm{P}=0.629)$.

Failure modes for representative specimens, the highest, middle and lowest bond strength values, for each location-grit combination are depicted in Table 3. Overall, the highest and middle SBS values showed mixedtype failures irrespective of the surface preparation or location of the bonding. The only two exceptions were the highest SBS values within each of these groups which showed cohesive failures. The lowest SBS values displayed predominantly adhesive failures.

\section{DISCUSSION}

This study evaluated the shear bond strength of specimens bonded to different crown regions of superficial bovine incisor dentin after preparation with 120-grit, 320 -grit or 600-grit SiC abrasive paper. Overall, high bond strength values were demonstrated irrespective of the crown location and surface preparation. The first null hypothesis was confirmed as no significant differences in

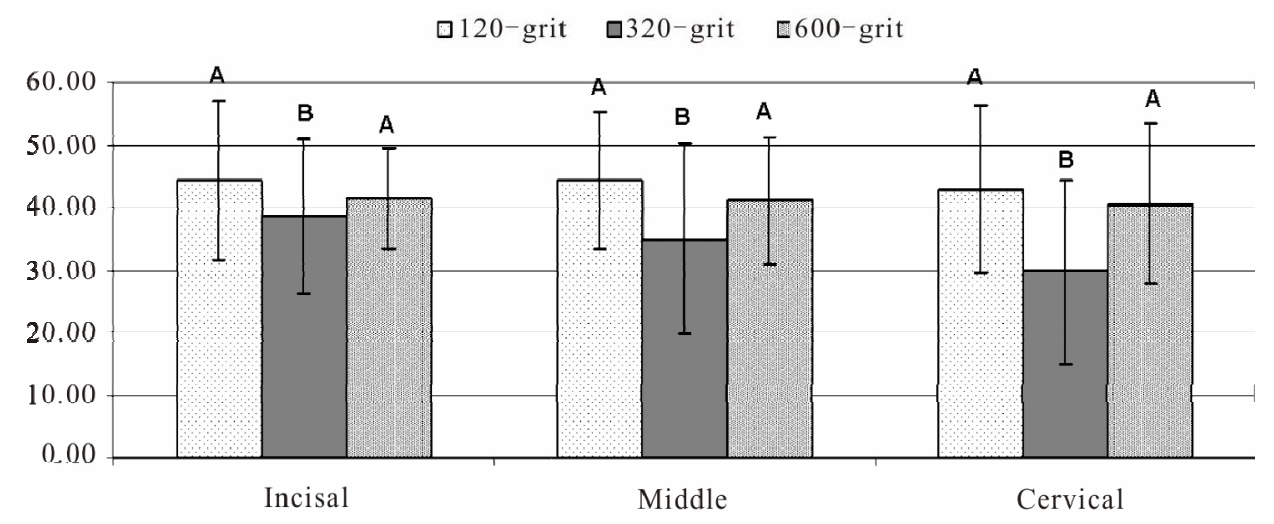

Figure 1. Mean shear bond strength values for the different location-grit combinations. Same letter indicates no significant differences between grits for each crown third. 
Table 1. Mean shear bond strength and standard deviation values for the different location-grit combinations.

\begin{tabular}{cccc}
\hline SBS MPa (SD) & 120-grit & 320-grit & 600-grit \\
\hline Incisal third & $44.4(12.7)$ & $38.6(12.3)$ & $41.4(7.9)$ \\
Middle third & $44.4(10.8)$ & $35.0(15.4)$ & $41.0(10.1)$ \\
Cervical third & $43.0(13.5)$ & $30.0(14.8)$ & $40.5(12.9)$ \\
\hline
\end{tabular}

bond strength values were shown for the different crown thirds. Despite the lack of significant differences, certain trends were observed indicating that orientation of the dentinal tubules may have had an effect on the bond strength results. Regardless of the surface preparation, the cervical third consistently showed the lowest SBS values and the middle and incisal thirds showed similar SBS values except for the groups prepared with 320-grit for which the incisal third displayed higher mean SBS value than that shown for the middle third. Similarly, a previous study evaluating shear bond strength to different crown regions demonstrated that, despite the absence of significant differences, a trend to higher values could be observed when bonding to the middle third of superficial bovine coronal dentin [25]. Hence, it appears that there could be an influence of the dentinal tubule orientation in the bond strength results that may only be detectable with a larger sample size. However, the sample size of twenty specimens per study group used in both of these studies doubles the sample size of ten commonly used in bond strength studies $[15,26]$. Based on these results, it may be safe to make the assumption that superficial bovine incisor dentin displays isotropic SBS properties and that provided that the same dentin depth is used, bonding can be performed to the different crown regions without a significant effect on the bond strength. The literature shows conflicting evidence as to the effect of dentinal tubule orientation in bond strength with studies showing a positive correlation between tubular orientation and bond strength results [27] and studies showing no correlation [28]. Histochemical and morphological studies have shown that human and bovine dentin are essentially the same [9-11]. Hence, the reported isotropic SBS behavior of bovine incisor dentin offers the added advantage of providing a much larger surface area for bonding compared to the use of human molars. Dentinal tubules are arranged in a radial pattern from the pulp chamber to the dentino-enamel junction (DEJ) and follow an "S" or zigzag course. Despite the zigzag course, dentinal tubules are oriented approximately at $90^{\circ}$ to the DEJ in superficial dentin irrespective of the crown third suggesting that different crown regions may exhibit similar mechanical properties [29].

The second null hypothesis was rejected as differences in SBS were observed when the surface was prepared with different grits of $\mathrm{SiC}$ abrasive paper. Higher bond strength results were obtained for 600- and 120-grit
Table 2. Two-way ANOVA results (DF: Degrees of freedom, SS: Sum of squares, MS: Mean Squares).

\begin{tabular}{cccccc}
\hline Source of Variation & DF & SS & MS & F & P \\
\hline Location & 2 & 429.149 & 214.574 & 1.381 & 0.254 \\
Grit & 2 & 2815.321 & 1407.661 & 9.062 & $<0.001$ \\
Location $\times$ Grit & 4 & 403.047 & 100.762 & 0.649 & 0.629 \\
Residual & 171 & 26561.583 & 155.331 & & \\
Total & 179 & 30209.100 & 168.76 & & \\
\hline
\end{tabular}

abrasive paper with both grits showing values statistically higher than those obtained with the 320 -grit group. Both viscosity of the adhesive and specimen's surface topography have been reported to be the main factors responsible for the nature and intimacy of the interactions taking place between the adhered and the adhesive [30]. Optibond Solo Plus is a single-component dental adhesive filled $15 \%$ by weight with $0.4 \mu \mathrm{m}$ barium aluminoborosilicate glass and silicon dioxide. The filler reinforces the hybrid zone but at the same time, makes the adhesive more viscous perhaps hindering its ability to flow into areas of compromised access. The success of adhesive interfaces with etch-and-rinse systems depends largely on the ability of the resin monomers to penetrate to the same depth to which demineralization has occurred creating a strong hybrid layer [31]. Studies have shown that greater surface roughness promotes wettability by producing greater surface area for bonding, and as a consequence, a stronger micro-mechanical bond can be obtained [19]. The results of our study challenge this assumption. Although the highest SBS values were observed when $\mu$ surfaces were prepared with 120 -grit, the second highest SBS results were obtained for the groups prepared with 600 -grit rather than 320 -grit. Interestingly, no significant differences were observed between SBS values obtained with 120 -grit and 600 -grit groups indicating no clinical difference between the two very different surface roughnesses.

Variations of micropore size are known to yield different surface topographies. According to the ISO standards for coated abrasives, the average grit size for the $\mathrm{SiC}$ abrasive papers are approximately: $25.8 \mu \mathrm{m}$ for 600 -grit, $46.2 \mu \mathrm{m}$ for 320 -grit, and $125 \mu \mathrm{m}$ for 120 -grit abrasive paper. (ISO 6344-1:1998) Figure 2 depicts scanning electron microscope images of dentin prepared with the three different grits of abrasive paper. The difference in surface topography obtained with each grit size relative to the smaller size of a dentinal tubule, which ranges between $1-2.5 \mu \mathrm{m}$, becomes evident in these images. A highly undulating surface topography was observed for the 120-grit abrasive paper. The pore size of 120-grit abrasive paper was approximately three times larger than that of 320-grit paper and five times larger than that of 600 -grit paper. Presumably this sur- 
Table 3. Mode of failure for representative SBS values for the incisal, middle and cervical crown thirds.

\begin{tabular}{|c|c|c|c|c|}
\hline SBS Value & Grit & Crown third & SBS (MPa) & Mode of Failure \\
\hline Highest & 120 & Incisal & 56.2 & Mixed \\
\hline Highest & 120 & Middle & 59.1 & Cohesive (dentin) \\
\hline Highest & 120 & Cervical & 58.5 & Mixed \\
\hline Highest & 320 & Incisal & 58.6 & Mixed \\
\hline Highest & 320 & Middle & 54.6 & Mixed \\
\hline Highest & 320 & Cervical & 55.2 & Mixed \\
\hline Highest & 600 & Incisal & 55.5 & Mixed \\
\hline Highest & 600 & Middle & 56.3 & Mixed \\
\hline Highest & 600 & Cervical & 56.8 & Mixed \\
\hline Middle & 120 & Incisal & 44.7 & Mixed \\
\hline Middle & 120 & Middle & 45.9 & Cohesive (dentin) \\
\hline Middle & 120 & Cervical & 42.2 & Mixed \\
\hline Middle & 320 & Incisal & 41.1 & Mixed \\
\hline Middle & 320 & Middle & 34.5 & Mixed \\
\hline Middle & 320 & Cervical & 30.5 & Mixed \\
\hline Middle & 600 & Incisal & 41.2 & Mixed \\
\hline Middle & 600 & Middle & 41.2 & Mixed \\
\hline Middle & 600 & Cervical & 42.8 & Mixed \\
\hline Lowest & 120 & Incisal & 27.7 & Mixed \\
\hline Lowest & 120 & Middle & 19.1 & Adhesive (DBA/composite) \\
\hline Lowest & 120 & Cervical & 7.7 & Adhesive (DBA/dentin) \\
\hline Lowest & 320 & Incisal & 16.1 & Adhesive (DBA/dentin) \\
\hline Lowest & 320 & Middle & 12.8 & Adhesive (DBA/dentin) \\
\hline Lowest & 320 & Cervical & 11.8 & Adhesive (DBA/dentin) \\
\hline Lowest & 600 & Incisal & 26.7 & Adhesive (DBA/dentin) \\
\hline Lowest & 600 & Middle & 17.5 & Adhesive (DBA/composite) \\
\hline Lowest & 600 & Cervical & 10.7 & Adhesive (DBA/composite) \\
\hline
\end{tabular}

DBA: Dentin Bonding Agent.

face topography allowed free flow of the filled viscous adhesive into the demineralized dentin matrix creating a strong micro-mechanical interlocking. This is evidenced by the highest bond strengths obtained with 120-grit paper. Conversely, 600-grit abrasive paper yielded the smoothest surface topography of all groups. However, the shallower less wavy surface obtained with this grit also allowed strong hybridization as evidenced by the high bond strength results observed for this group. The observed lowest bond strength results obtained with 320-grit abrasive paper may have been the result of an incomplete infiltration of the adhesive monomers into the demineralized dentin matrix. Although the thickness of the smear layer after preparation with the different abrasive papers was not evaluated as a part of this study, it may have played a role on the observed bond strength results. Differences in smear layer thickness have been reported to affect dentin permeability $[23,24]$ and bond strength of adhesives [24]. The lower bond strengths obtained with 320-grit abrasive paper may have been the combined result of a rather thick smear layer which was perhaps incompletely removed during acid etching procedures.

It is interesting that ISO Standards for bond strength testing recommend surface preparation of the substrate with 600-grit abrasive paper. While good bond strength results were obtained when the surface was prepared with 600-grit paper, we argue that the surface roughness obtained with this type of surface finish may not always be representative of the real clinical situation when the tooth structure is prepared with different types of carbide or diamond burs. A study compared dentinal surfaces prepared with ether a carbide bur or SiC papers and demonstrated that preparation of the surface with 320grit paper followed by a 254-carbide bur most closely resembled the finish obtained clinically after cavity preparation with a tungsten carbide bur [20].

\section{Analysis of the Mode of Failure}

Our study also evaluated the failure mode for representative specimens for each study group. Although no corre- 

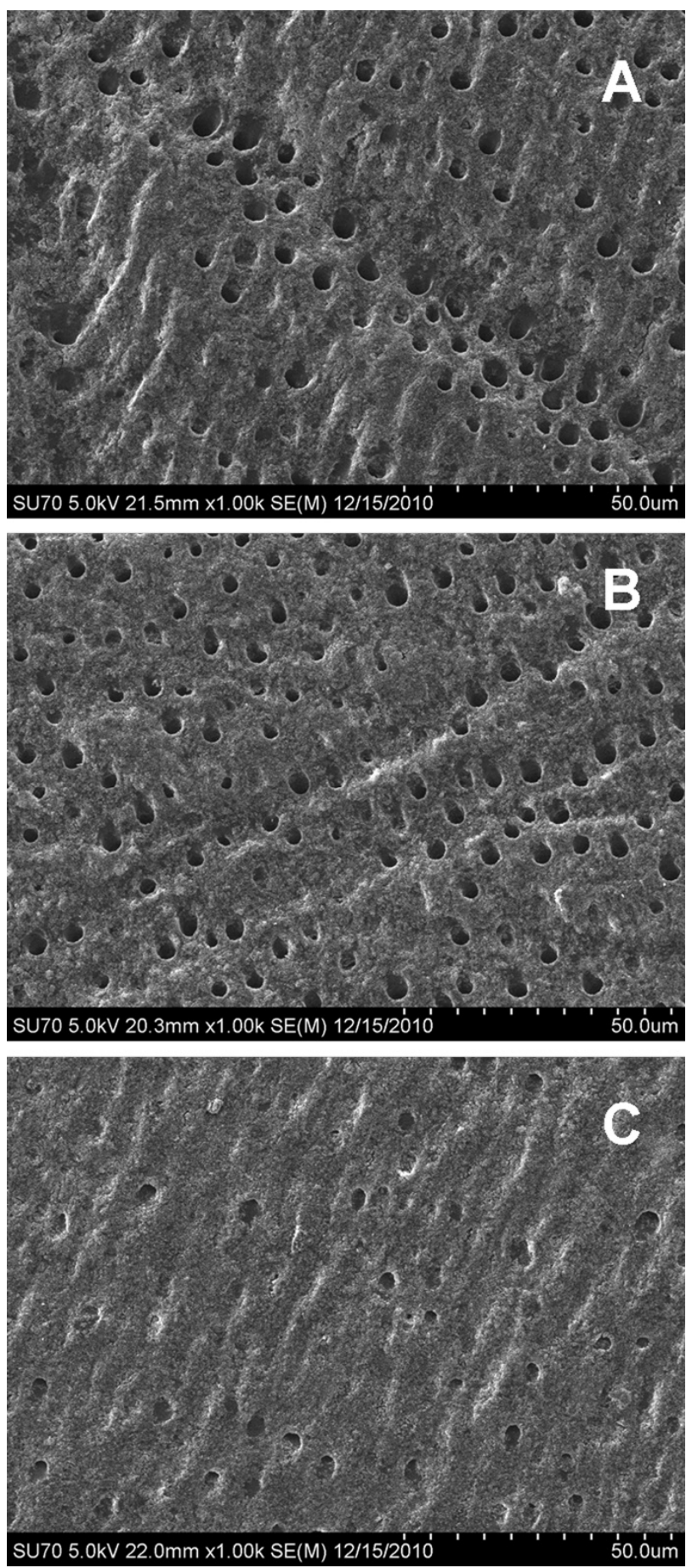

Figure 2. Scanning electron microscope images in secondary electron mode display surface topography obtained with the different grits of $\mathrm{SiC}$ abrasive paper. (A) 120-grit; (B) 320-grit; (C) 600-grit.

lation was observed between bond strength results and surface preparation or location of the bonding, a strong correlation between SBS values and mode of failure was evident with lower SBS values showing predominantly "adhesive" failures between dentin and the adhesive resin and higher SBS values showing "mixed" failure through the body of the adhesive interface. It appears then that the entire adhesive interface assembly behaves much stronger when acting together as a single body rather than as separate layers. This suggests that the intimacy of the micro-mechanical adhesion between the adhesive resin and the partially demineralized collagen network is such that it surpasses the cohesive strength of each of its individual components and the adhesive strength between the different interfacial layers. Our results are in agreement with studies that have shown a strong positive correlation between strength values and the area of cohesive failure in resin observed in mixed failures has also been demonstrated [2,32].

Nevertheless, there is still minimal understanding regarding the mechanism by which adhesive interfaces fail. The presence of "mixed" failures where there is a combination of cohesive and adhesive failures within the same bonded area, and how an adhesive bond transitions from a strong bond which exhibits cohesive failure to a weak bond which exhibits adhesive failure should be further studied. Failure of adhesive interfaces may be explained by the parameters involved in crack propagation whereby a crack propagates from a critical size flaw found in an area subjected to high stresses [33]. Scanning electron microscope imaging revealed that most failures for the high bond strength specimens were of the mixed type; that is the loading device caused the assembly to split in a cohesive-adhesive fashion, making it a challenge to identify the true plane of separation. A number of aspects play a role in the strength, adhesive or cohesive, of adhesives interfaces making it a challenge to understand its complex nature and behavior when subjected to stress during bond strength testing [4]. This great complexity of the adhesive interface previously reported in the literature [2,34]. The mechanics of the strength testing itself is complex in nature with concentration of forces at the point where the cross-head first contacts the interface. The particular test assembly used in our study, the Ultradent notched rod, uses a larger contact area between the composite and the loading device which theoretically allows better stress distribution than other commonly used assemblies such as the knife-edge chiesel which concentrates stress at a single load application point $[35,36]$. The complex fracture mechanics of adhesive interfaces may be explained by the intricate nature of the test itself. When the cross-head device first contacts the bonded interface, there is a concentration of forces that accumulate on the site where the cross-head first contacts the interface. These forces accumulate until they reach a point of failure which is reported as a nominal bond strength value. Subsequent propagation of the initial fracture will occur cohesively though one of the substrates or adhesively through the 
tooth-adhesive or adhesive-composite interfaces, and it will depend on aspects such as the original failure point, the physical and mechanical properties of the individual materials involved in the interface as well as their properties and behavior when combined as a single body.

\section{CONCLUSIONS}

Within the limitations of this study, the following conclusions may be drawn:

- The anisotropic behavior of superficial bovine dentin relative to bond strength was not confirmed.

- Regardless of the location, 320-grit consistently showed the lowest SBS and 120-grit showed the highest SBS indicating that different surface grit preparations have an effect on the in-vitro dentin bond strength.

- A correlation between the SBS values and the failure mode was observed. Specimens with high bond strengths showed predominantly "mixed-type" failures and specimens with low bond strengths showed mainly "adhesive" failures.

\section{ACKNOWLEDGEMENTS}

The authors would like to thank Mr. Peter Bush for his assistance with the SEM imaging.

\section{REFERENCES}

[1] De Munck, J., Van Landuyt, K., Peumans, M., Poitevin, A., Lambrechts, P., Braem, M. and Van Meerbeek, B. (2005) A critical review of the durability of adhesion to tooth tissue: Methods and results. Journal of Dental Research, 84, 118-132. doi:10.1177/154405910508400204

[2] Leloup, G., D’Hoore, W., Bouter, D., Degrange, M. and Vreven, J. (2001) Meta-analytical review of factors involved in dentin adherence. Journal of Dental Research, 80, 1605-1614. doi:10.1177/00220345010800070301

[3] Pashley, D.H. and Carvalho, R.M. (1997) Dentine permeability and dentine adhesion. Journal of Dentistry, 25, 355-372. doi:10.1016/S0300-5712(96)00057-7

[4] Pashley, D.H., Sano, H., Ciucchi, B., Yoshiyama, M. and Carvalho, R.M. (1995) Adhesion testing of dentin bonding agents: A review. Dental Materials, 11, 117-125. doi:10.1016/0109-5641(95)80046-8

[5] Rueggeberg, F.A. (1991) Substrate for adhesion testing to tooth structure-Review of the literature. Dental Materials, 7, 2-10. doi:10.1016/0109-5641(91)90017-S

[6] Burke, F.J., Hussain, A., Nolan, L. and Fleming, G.J. (2008) Methods used in dentine bonding tests: an analysis of 102 investigations on bond strength. European Journal of Prosthodontics and Restorative Dentistry, 16, 158-165.

[7] Marshall Jr., G.W., Marshall, S.J., Kinney, J.H. and Balooch, M. (1997) The dentin substrate: Structure and properties related to bonding. Journal of Dentistry, 25, 441-458. doi:10.1016/S0300-5712(96)00065-6
[8] Tagami, J., Tao, L., Pashley, D.H. and Horner, J.A. (1989) The permeability of dentine from bovine incisors in vitro. Archives of Oral Biology, 34, 773-777. doi:10.1016/0003-9969(89)90027-7

[9] Schilke, R., Lisson, J.A., Bauss, O. and Geurtsen, W. (2000) Comparison of the number and diameter of dentinal tubules in human and bovine dentine by scanning electron microscopic investigation. Archives of Oral Biology, 45, 355-361. doi:10.1016/S0003-9969(00)00006-6

[10] Nakamichi, I., Iwaku, M. and Fusayama, T. (1983) Bovine teeth as possible substitutes in the adhesion test. Journal of Dental Research, 62, 1076-1081. doi:10.1177/00220345830620101501

[11] Reis, A.F., Giannini, M., Kavaguchi, A., Soares, C.J. and Line, S.R. (2004) Comparison of microtensile bond strength to enamel and dentin of human, bovine, and porcine teeth. The Journal of Adhesive Dentistry, 6, 117-121.

[12] Saunders, W.P. (1988) The shear impact retentive strengths of four dentine bonding agents to human and bovine dentine. Journal of Dentistry, 16, 233-238. doi:10.1016/0300-5712(88)90080-2

[13] Reeves, G.W., Fitchie, J.G., Hembree Jr., J.H. and Puckett, A.D. (1995) Microleakage of new dentin bonding systems using human and bovine teeth. Operative Dentistry, 20, 230-235.

[14] Schüpbach, P., Krejci, I. and Lutz, F. (1997) Dentin bonding: Effect of tubule orientation on hybrid-layer formation. European Journal of Oral Sciences, 105, 344352. doi:10.1111/j.1600-0722.1997.tb00251.x

[15] Inoue, T., Takahashi, H. and Nishimura, F. (2002) Anisotropy of tensile strengths of bovine dentin regarding dentinal tubule orientation and location. Dental Materials Journal, 21, 32-43. doi:10.4012/dmj.21.32

[16] Mowery Jr., A.S., Parker, M. and Davis, E.L. (1987) Dentin bonding: The effect of surface roughness on shear bond strength. Operative Dentistry, 12, 91-94.

[17] Gupta, R. and Tewari, S. (2006) Effect of rotary instrumentation on composite bond strength with simulated pulpal pressure. Operative Dentistry, 31, 188-196. doi:10.2341/05-4

[18] Wahle, J.J. and Wendt Jr., S.L., (1993) Dentinal surface roughness: A comparison of tooth preparation techniques. Journal of Prosthetic Dentistry, 69, 160-164. doi:10.1016/0022-3913(93)90135-B

[19] Ayad, M.F., Johnston, W.M. and Rosenstiel, S.F. (2009) Influence of dental rotary instruments on the roughness and wettability of human dentin surfaces. Journal of Prosthetic Dentistry, 102, 81-88. doi:10.1016/S0022-3913(09)60114-1

[20] McInnes, P.M., Wendt Jr., S.L., Retief, D.H. and Weinberg, R. (1990) Effect of dentin surface roughness on shear bond strength. Dental Materials, 6, 204-207. doi:10.1016/0109-5641(90)90031-9

[21] Hosoya, Y., Shinkawa, H., Suefiji, C., Nozaka, K. and Garcia-Godoy, F. (2004) Effects of diamond bur particle size on dentin bond strength. American Journal of Dentistry, 17, 359-364. 
[22] Peerzada, F., Yiu, C.K., Hiraishi, N., Tay, F.R. and King, N.M. (2010) Effect of surface preparation on bond strength of resin luting cements to dentin. Operative Dentistry, 35, 624-633. doi:10.2341/09-379-L

[23] Tagami, J., Tao, L., Pashley, D.H., Hosoda, H. and Sano, H. (1991) Effects of high-speed cutting on dentin permeability and bonding. Dental Materials, 7, 234-239. doi:10.1016/S0109-5641(05)80021-1

[24] Ogata, M., Harada, N., Yamaguchi, S., Nakajima, M. and Tagami, J. (2002) Effect of self-etching primer vs phosphoric acid etchant on bonding to bur-prepared dentin. Operative Dentistry, 27, 447-454.

[25] Sabatini, C. and Andreana, S. (2013) Isotropic shear bond strength behavior of superficial bovine dentin: A pilot study. Open Journal Stomatology, 3, 1-7. doi:10.4236/ojst.2013.31001

[26] Braga, R.R., Meira, J.B., Boaro, L.C. and Xavier, T.A. (2010) Adhesion to tooth structure: A critical review of "macro" test methods. Dental Materials, 26, e38-e49. doi:10.1016/j.dental.2009.11.150

[27] Watanabe, L.G., Marshall, J.G.W. and Marshall, S.J. (1996) Dentin shear strength: Effects of tubule orientation and intratooth location, Dental Materials, 12, 109-115. doi:10.1016/S0109-5641(96)80077-7

[28] Phrukkanon, S., Burrow, M.F. and Tyas, M.J. (1999) The effect of dentine location and tubule orientation on the bond strengths between resin and dentine. Journal of Dentistry, 27, 265-274. doi:10.1016/S0300-5712(98)00060-8

[29] Kinney, J.H., Marshall, S.J. and Marshall, G.W. (2003) The mechanical properties of human dentin: A critical review and re-evaluation of the dental literature. Critical
Reviews in Oral Biology \& Medicine, 14, 13-29. doi: $10.1177 / 154411130301400103$

[30] Eick, J.D., Johnson, L.N., Fromer, J.R., Good, R.J. and Neumann, A.W. (1972) Surface topography: Its influence on wetting and adhesion in a dental adhesive system. Journal of Dental Research, 51, 780-788. doi:10.1177/00220345720510031401

[31] Nakabayashi, N. and Takarada, K. (1992) Effect of HEMA on bonding to dentin. Dental Materials, 8, 125130. doi:10.1016/0109-5641(92)90067-M

[32] Al-Assaf, K., Chakmakchi, M., Palaghias, G., Karanika-Kouma, A. and Eliades, G. (2007) Interfacial characteristics of adhesive luting resins and composites with dentine. Dental Materials, 23, 829-839. doi:10.1016/j.dental.2006.06.023

[33] Loughran, G.M., Versluis, A. and Douglas, W.H. (2005) Evaluation of sub-critical fatigue crack propagation in a restorative composite. Dental Materials, 21, 252-261. doi:10.1016/i.dental.2004.04.005

[34] Nakabayashi, N., Nakamura, M. and Yasuda, N. (1991) Hybrid layer as a dentin-bonding mechanism. Journal of Esthetic and Restorative Dentistry, 3, 133-138. doi:10.1111/j.1708-8240.1991.tb00985.x

[35] De Hoff, P.H. Anusavice, K.J. and Wang, Z. (1995) Three-dimensional finite element analysis of the shear bond test. Dental Materials, 11, 126-131. doi:10.1016/0109-5641(95)80047-6

[36] Pecora, N., Yaman, P., Dennison, J. and Herrero, A. (2002) Comparison of shear bond strength relative to two testing devices. Journal of Prosthetic Dentistry, 88, 511515. doi:10.1067/mpr.2002.129063 